Service social

\title{
Les tâches, un instrument privilégié du changement
}

\section{Denise Robitaille et Christian Côté}

Volume 43, numéro 3, 1994

Intervention individualisée et empowerment

URI : https://id.erudit.org/iderudit/706669ar

DOI : https://doi.org/10.7202/706669ar

Aller au sommaire du numéro

Éditeur(s)

École de service social de l'Université Laval

ISSN

1708-1734 (numérique)

Découvrir la revue

Citer cet article

Robitaille, D. \& Côté, C. (1994). Les tâches, un instrument privilégié du changement. Service social, 43(3), 79-94. https://doi.org/10.7202/706669ar

\section{Résumé de l'article}

L'article présente la notion de tâche et les fonctions liées aux tâches. Il en explique le fondement : l'apprentissage, le développement de la motivation, l'apport d'inputs nouveaux et l'effet sur la relation. Il précise les types de tâches : observation, réflexion, action directe et action indirecte ou paradoxale. Enfin, à partir de leurs expérimentations, les auteurs développent les modalités de sélection, de prescription et de retour sur les tâches, avec exemples. 


\section{Les tâches, un instrument privilégié de changement}

DENISE ROBITAILLE Centre de consultation conjugale et familiale de Québec

CHRISTIAN CÔTÉ Professeur

École de service social, Université Laval

Tous les intervenants, en situation de problèmes sociaux ou interpersonnels, demandent à un moment ou à un autre à leurs clients des activités, soit pour obtenir des informations, soit pour acquérir des connaissances, soit pour expérimenter des modes nouveaux de comportements. Ces activités sont des tâches. L'approche planifiée systémique à court terme en a fait son instrument principal d'intervention. Nous avons constaté que cette technique est souvent mal utilisée, et qu'elle est donc alors inefficace. L'article qui suit présente les résultats de nos réflexions et de nos expérimentations ainsi que les expériences de cliniciens-chercheurs avec qui nous avons eu le plaisir de travailler. II propose une définition acceptée par les auteurs reconnus pour leur utilisation de la tâche, I'analyse raisonnée sous-jacente à cette technique, les types de tâches, leur utilisation et les pièges à éviter. 


\section{CE QU'EST LA TÂCHE}

Toutes les approches utilisent la prescription d'activités: la psychanalyse requiert que le patient note ses rêves, le béhaviorisme organise des séquences rigoureuses de comportements et la gestalt demande de s'adresser de façon précise à une chaise apparemment vide. Le principal chercheur en service social sur l'intervention centrée sur la tâche est William Reid qui, avec Laura Epstein, a dirigé et inspiré de nombreuses équipes de recherche et de développement des modalités d'intervention utilisant cette technique de façon systématique. Mathilde Du Ranquet I'a traduit et adapté au milieu français. Au Québec, des équipes de la Société de service social aux familles de Montréal, du Service familial de Québec, de l'École de service social de l'Université Laval, de même que le Centre de consultation conjugale et familiale de Québec, ont repris et développé cette approche.

La tâche paradoxale a été élaborée par Milton Ericson, par Jay Haley à Philadelphie et à Washington ainsi que par l'équipe du Mental Research Institute de Palo Alto.

Les recherches (Reid et Epstein, 1977 ; Epstein, 1980 ; Fortune, 1985) indiquent que l'utilisation systématique de la tâche contribue à atteindre de façon significative l'objectif non seulement de la tâche, mais de l'intervention, avec différentes catégories de clients: enfants, adolescents, adultes, personnes âgées, couples, familles, et cela dans toutes les problématiques. Fisher (1980) a constaté que les clients connaissaient plus de changements lorsqu'ils avaient à réaliser des activités en lien avec leurs problèmes.

Tous les auteurs s'entendent pour définir la tâche comme une activité clairement identifiée, limitée et structurée, comprenant quelques actions réalisées en continuité, que le système-client fait à la demande du travailleur social, en vue d'atteindre un objectif immédiat. C'est une unité structurée de travail (Reid, 1985) que I'intervenant organise à un moment précis, dans le but de libérer des informations et d'élaborer une façon nouvelle de réagir à une situation habituelle.

\section{SA JUSTIFICATION}

Le raisonnement sous-tendant la tâche comprend quatre éléments. Le premier est que les approches phénoménologiques, centrées sur l'observation des phénomènes vérifiables, postulent que le changement se réalise par les actions et ne se manifeste réellement que dans les comportements. Il se fonde sur les théories de l'apprentissage: 
le changement se réalise par l'action plus que par la parole. Cela semble aller contre la culture occidentale "officielle» qui insiste sur la parole comme instrument de transmission des informations et d'apprentissage. De fait, pour la plupart des personnes, le langage verbal est le moyen le plus approprié pour échanger des messages avec précision. Mais ceux qui s'occupent de formation savent que les apprentissages réels requièrent la pratique, en plus de la connaissance ; tant qu'une personne $\mathrm{n}^{\prime}$ a pas expérimenté et répété des gestes, les messages reçus demeurent une théorie, ils $\mathrm{n}^{\prime}$ " informent » pas, et les habiletés sont connues, non acquises. Plusieurs théoriciens peuvent décrire des activités de façon précise, mais sans pouvoir les réaliser habilement: ils ne les ont pas pratiquées suffisamment. La plupart des clients savent exactement ce qu'ils doivent faire, mais sans réussir à le faire. Nous pouvons le leur expliquer de nouveau, souvent mieux qu'eux, et nous avons le même résultat: une connaissance qui ne débouche pas sur le changement désiré.

Il est donc nécessaire de susciter des gestes qui permettront l'apprentissage. Minuchin (1981) parle d'enactment, ce que nous traduisons par scénario. Haley (1974) utilise le terme de directives. Minuchin et Montalvo (1966) recommandent, dans les situations où la structure des relations et des communications est chaotique, et spécialement lorsque les frontières sont enchevêtrées, de faire agir plus que de faire parler: les informations sont reçues plus clairement sans être immédiatement déformées. Les actions contournent alors les obstacles posés par les patterns défensifs.

Un deuxième élément est l'augmentation de la confiance et de la motivation au changement résultant des tâches bien prescrites et réussies. Un objectif de la tâche est de placer le client en situation de réussite, si petite soit cette réussite. La précision et la faisabilité des actions à faire diminuent la crainte et offrent de l'espoir. L'expérimentation d'une façon alternative et efficace de réagir dans des situations vécues jusque-là comme sans issue amène de l'espoir en une amélioration. Le succès dans les petites actions développe la confiance que d'autres petites actions pourront être faites et que finalement l'objectif sera atteint. De plus, toute action que le client accepte de poser l'engage davantage, sans qu'il en soit conscient.

Lorsque plusieurs personnes participent à la tâche, elles y expérimentent de la coopération, ce qui est souvent une découverte pour celles qui vivent une situation de conflits constants; cette expérience peut ensuite être utilisée dans d'autres situations.

Un troisième facteur réside dans le processus d'intervention: les tâches constituent des inputs nouveaux qui déséquilibrent les 
patterns connus par les client et facilitent l'action des forces de changement. Notre postulat est que toute personne a des forces et des compétences pour faire face aux situations qu'elle rencontre, et que des patterns appris l'empêchent d'avoir accès à ses ressources ou de les utiliser. L'input apporté par la tâche ouvre à une utilisation différente de ces forces, en les guidant par les directives dans une piste nouvelle.

Haley propose un quatrième élément, assez surprenant à première vue: la tâche augmente la relation avec l'intervenant, donc le pouvoir que le client lui reconnaît. Les tâches réalisées entre les entrevues maintiennent l'existence du système de changement formé par le t.s. et le client même lorsqu'il n'est plus avec le travailleur social. Elles constituent un rappel que le client désire un changement et s'y est engagé. Elles peuvent également diminuer certaines tensions par le rappel que le travailleur social est avec lui dans cette recherche de la situation désirée.

Plusieurs s'opposent aux tâches en disant qu'il s'agit de conseils et que nous $\mathrm{n}^{\prime}$ avons pas à en donner, ou que les suggestions venant de l'extérieur du système concerné ne sont pas valables pour lui. Notre réponse est que la tâche n'est pas présentée comme un conseil, mais comme une action visant un apprentissage précis vers I'atteinte de la situation désirée. De plus, les tâches sont choisies à partir des propos et des recommandations des clients. La planification et la prescription des tâches reposent sur la compétence de l'intervenant et la demande faite par le client. Elles s'inscrivent dans la poursuite du contrat passé entre le travailleur social et le client. La directivité s'exerce alors suivant des règles claires et souples, dans l'écoute de ce que le client nous dit: les frontières sont alors saines. Une étude menée en 1969 par Reid et Shapiro (citée par Du Ranquet, 1991) indique que le manque de conseils constitue le principal reproche des clients : ils veulent avoir l'opinion et les recommandations d'une personne jugée experte.

Il est toutefois important que les conseils s'insèrent dans un processus d'apprentissage, qu'ils respectent son unicité et tiennent compte de son rythme de changement. Autrement, les conseils des intervenants ne se distinguent pas de ceux que toute personne peut fournir avec son expérience.

\section{TYPES DE TÂCHES}

Reid (1985) distingue les tâches générales et les tâches particulières. Les tâches générales demandent que des gestes soient faits pour 
arriver à un but, mais sans préciser les actions requises. L'intervenant devra déterminer les acteurs, le thème, le but, la limite de temps, sans préciser les actions à prendre pour la réussir. Par exemple, nous pourrions demander à des parents (les acteurs) d'arriver à une entente (le but) sur les heures de rentrée de leur adolescente (le thème) d'ici la prochaine rencontre (la limite de temps). Le client a toute la latitude de déterminer les actions à prendre pour réussir la tâche, à partir de ses connaissances et de ses compétences. On peut dire à un parent de contrôler durant l'entrevue son enfant hors contrôle, sans spécifier comment y arriver.

Les tâches générales demandent peu de temps à prescrire, mais sont plus difficiles à réussir parce qu'elles sont imprécises. Nous avons constaté que la plupart des tâches non réalisées sont des tâches générales. Nous proposons de les utiliser davantage en fin du processus d'intervention, lorsque le client a fait plusieurs apprentissages auxquels il peut se référer.

$C^{\prime}$ est pourquoi nous favorisons les tâches particulières. Nous y distinguons trois types d'activités:

- Les tâches d'observation mettent le client en situation de recueillir des informations exactes pour mieux cerner la situation-problème, les difficultés rencontrées, les attitudes problématiques, mais aussi les forces, les succès, les «exceptions» aux problèmes, les solutions existantes. Le client recueille lui-même ces informations, et peut prendre conscience de leur valeur. Elles sont utilisées le plus souvent en cours d'exploration pour alimenter la collecte de données, et dans les premières étapes d'un changement pour mieux cerner les obstacles et les ressources. Une observation "guidée» de la situation facilite l'identification des différents participants dans le problème et leur implication. Le problème ne peut plus demeurer «à l'extérieur » de la personne qui fait une demande.

L'observation peut porter sur des comportements précis, leur fréquence, les séquences d'un pattern, des pensées négatives ou positives, etc., sur les réussites des clients ou d'autres personnes dans une situation du même type, des pensées positives, etc. Elles sont de l'ordre des faits, des agirs.

La prescription d'une tâche d'observation sera détaillée: déterminer une situation où l'observation se fera, prendre note des actions, des propos des autres et aussi des siens, de I'intonation des paroles, de la durée de l'action et de la façon dont elle se termine. 
Nous avons constaté à maintes reprises que les tâches d'observation amènent des modifications positives dans la situation sans que ces changements aient été prescrits.

- Les tâches de réflexion ont pour objet d'amener le systèmeclient à faire un arrêt pour prendre conscience de ce qu'il vit ou désire réellement, établir ses priorités, organiser et intégrer des connaissances, clarifier des ambiguïtés. Comme les tâches d'observation, elles fournissent de précieuses informations sur les croyances, les valeurs, les priorités, les désirs.

Il est essentiel de prescrire une tâche de réflexion pour déterminer les changements désirés. Elle prépare le contrat et la planification des stratégies d'interventions. Plus la tâche de réflexion en rapport avec la demande sera spécifique, plus les priorités et les interventions seront pertinentes.

Elles sont souvent jointes à des tâches d'observation en phase d'exploration et dans les premières étapes de la réalisation du changement désiré. Nous les utilisons également en fin d'intervention, dans le bilan, pour aider le client à s'approprier le crédit des changements et à comprendre la démarche poursuivie pour les réaliser. Elles lui permettent de mieux identifier les activités qui ont contribué à la réalisation des changements, ce qui l'aidera à les utiliser en d'autres occasions.

Elles peuvent être des lectures pour acquérir des connaissances, des écrits sur ses perceptions, des listes de succès ou de problèmes à travailler, de priorités, de questions sans réponses, des analyses, des récits de situations passées ou de projections futures. Elles sont de l'ordre du cognitif, du réfléchi.

Une tâche de réflexion pour l'élaboration des changements visés est la visualisation: nous demandons au client de se projeter dans le futur, à un moment où l'objectif général sera atteint, et d'identifier ses nouvelles compétences: "Je saurai que j'ai atteint l'objectif quand je serai capable de... ». Ces compétences doivent porter sur des actions: "...capable de contacter des amis pour faire une sortie», et non sur un état: " ...capable $d^{\prime}$ être heureux ».

Nous utilisons de plus en plus des tâches d'observation et de réflexion, et nous constatons que les changements visés sont atteints plus rapidement. Notre hypothèse est que plus le client participe à l'élaboration des changements visés, plus il se mobilise, et que l'observation et la réflexion avant de passer à 
I'action permettent d'apprivoiser des changements perçus d'abord comme difficiles.

- Les tâches d'actions directes actualisent le développement $d^{\prime}$ 'une perception nouvelle, d'un apprentissage spécifique, d'une compétence, ou la réalisation d'un changement limité. Elles sont présentées en détail, de façon logique, et portent sur une action précise. Elles se situent dans des stratégies directes où les modalités et l'effet de la tâche sont clairs et compréhensibles par le système-client.

Les tâches d'actions directes se situent dans les stratégies d'intervention pour réaliser les changements visés. Cela implique que le contrat est établi et que le système-client a clairement donné son accord pour être guidé dans le processus de changement. S'il n'y a pas encore de contrat ou entente claire de travail commun, donc pas de consensus sur le changement et le rôle de l'intervenant, elles peuvent être utilisées pour vérifier la capacité de changement. Nous disons clairement: "Fais ce que tu peux dans cette activité, nous regarderons ensemble ce que tu as réussi à faire", afin que la non-réalisation ou la réalisation partielle ne soient pas perçues comme un échec.

Ces tâches comprennent des activités personnelles ou transactionnelles, faciles ou difficiles, sérieuses ou faites en jeu. Elles font appel à la créativité de l'intervenant, qui utilise les résultats des tâches d'observation et de réflexion qui ont précédé.

- Les tâches d'actions indirectes ou paradoxales visent à contourner une "résistance» au changement que le système-client dit vouloir réaliser et pour lequel il demande notre aide. Elles ne semblent pas logiques et visent à provoquer un déséquilibre, une confusion. Le but est de permettre aux forces de changement du système de pouvoir poser l'action qui amènera le changement désiré et craint en même temps. La compétence que le client y développe ne se situe pas à un niveau rationnel, logique, et ne peut habituellement pas être répétée dans des situations semblables, tant qu'il ne comprend pas que ce soit son pattern habituel qui bloquait ses efforts.

Les principales tâches indirectes ou paradoxales sont la prescription du problème, faire semblant d'avoir le problème, reconnaître que le problème présenté continue à être présent, 
mais en demandant de faire comme s'il n'était pas présent, prescrire le problème en y changeant un élément, prescrire une tâche à côté du problème en assurant qu'elle ne réglera pas le problème, reconnaître que le problème ne peut être réglé immédiatement et proposer une tâche "en attendant ».

On peut y inclure les tâches métaphoriques, qui consistent en une tâche sur un thème lié au problème, mais en n'exprimant pas le lien et même le niant. Par exemple, on peut donner à des enfants qui ne peuvent exprimer des sentiments positifs la tâche de donner des soins chaleureux à un animal, chacun son tour, suivant un ordre établi et appliqué par les parents.

Nous pouvons utiliser ces tâches avant que le contrat soit formellement passé lorsque nous constatons que le systèmeclient désire un changement, mais connaît des obstacles majeurs internes, ou lorsqu'il demande un service mais refuse tout ce que nous proposons, ou lorsqu'il est un spécialiste des demandes de services inefficaces. Nous nous appuyons sur le fait qu'il a fait une première demande de service, et que ses patterns bloquent la demande qu'il a lui-même faite; agir autrement signifierait d'après nous demander qu'il règle d'abord son problème pour que nous l'aidions ensuite à le régler. Et nous soulignons qu'il peut en sortir à n'importe quel moment.

Nous les utilisons surtout après le contrat, lorsque nous constatons qu'il a de la difficulté à réaliser le changement demandé, ou qu'il résiste inutilement à une stratégie directe même s'il a donné son accord. La tâche paradoxale se fonde alors sur le consentement du système-client à ce que nous le guidions dans le cheminement vers I'objectif établi.

\section{UTILISATION DE LA TÂCHE}

\section{La sélection}

Comment choisir l'activité qui sera acceptée et efficace? Les préalables chez les intervenants sont un solide bon sens associé à des connaissances théoriques et méthodologiques approfondies et à une créativité active. Nous avons rencontré des intervenants qui les réussissent dès le début de leurs interventions: un talent personnel peut exister. 
II n'existe pas de guide précis sur la sélection des tâches, mais les points suivants se sont révélés utiles. S'il est nécessaire de faire confiance à sa créativité - donc la développer -, il est également important de l'encadrer par des connaissances personnelles et professionnelles. Il est bon de connaître son style d'intervenant: s'il est très prudent ou très rationnel, $s^{\prime}$ efforcer $d^{\prime} y$ ajouter une pincée de fantaisie et d'humour dans le choix des tâches; s'il est très intense, l'alléger; et s'il est fantaisiste, prendre le temps de planifier, par respect pour le client et par prudence pour son propre avenir professionnel.

Il faut oser: la prudence est la vertu de l'action, aimait rappeler le père Georges-Henri Lévesque. Après avoir étudié soigneusement la situation, I'intervenant doit oser essayer, en se souvenant que le système-client a ses mécanismes de digestion et de protection. Ne pas avoir peur du ridicule dans les tâches paradoxales et se protéger en annonçant que cela peut avoir l'air bizarre.

Ce sont surtout les connaissances professionnelles qui peuvent nous guider. Du Ranquet souligne que la tâche est une réponse à ce que la situation exige; il faut donc comprendre la situation et les patterns de fonctionnement, c'est-à-dire les faits et leur signification pour les personnes en cause, les patterns et leurs effets, les implications pour le fonctionnement du système. Cette connaissance du client nous aide à anticiper sa capacité d'accomplir la tâche prescrite.

La connaissance de la signification attribuée aux faits par le client est essentielle, car l'input nouveau doit être recevable par le système. La tâche est choisie d'après notre connaissance de ce qui convient aux capacités et aux modes de fonctionnement des personnes en cause; de ce qui est recevable, acceptable et utilisable; de ses capacités d'ingestion: si la bouchée est trop grosse, il s'étouffera ou la recrachera! C'est pourquoi nous recommandons de diviser par deux la première tâche choisie; et, si l'intervenant est «actif», de la diviser par quatre.

Pour déterminer le type de tâche, il est important de bien nous situer à l'étape du processus où nous nous trouvons, et le type de tâche qui y correspond. Et il est essentiel d'avoir une hypothèse pour organiser l'activité: si tel changement se produit dans cette situation immédiate ou cette étape, cela aura tel effet sur le changement plus vaste poursuivi. L'hypothèse devient: si le client fait tel apprentissage, ou développe telle perception nouvelle, ou se relie de telle façon nouvelle avec les personnes dans la situation, le changement immédiat désiré pourra se produire. Nous cherchons alors quelle activité permettra au client de réaliser l'apprentissage mentionné. 
Plusieurs intervenants font eux-mêmes le choix des tâches, en fonction des propos et des façons d'agir des clients. D'autres les font participer au choix et ne commencent pas en faisant des suggestions, mais en demandant ce que les clients pensent être valable pour atteindre l'objectif visé. Nous pouvons alors utiliser les techniques de la question-miracle: si par miracle le problème disparaissait, que ferais-tu de différent? ou celles de la baguette magique: si tu avais une baguette magique, quel moyen voudrais-tu avoir pour...? ou encore des questions circulaires (voir l'article de Thérèse Lane dans ce numéro).

Le choix se fait alors en tenant compte du langage du client, de ses images, de ses activités et de ses talents; d'après l'équipe du Mental Research Institute de Palo Alto (Fisch, Weakland et Segal, 1981), la tâche est un recadrage agi, où la personne ne fait que vérifier une idée que nous lui avons présentée à partir de ses propres demandes.

\section{La prescription}

Le terme "prescription» est fort, mais approprié. Il est nécessaire que le système-client perçoive clairement que le travailleur social est compétent et engagé, qu'il sait ce qu'il demande et pourquoi il le fait ; sinon, il n'a pas l'autorité pour demander de faire un geste (Haley, 1974). Nous croyons que la tâche est mieux réussie lorsqu'elle est particulière et que la formulation est claire, détaillée et directe. Les verbes sont affirmatifs, non conditionnels: les «j'aimerais » cèdent la place aux " $C^{\prime}$ 'est important d'ici notre prochaine rencontre de...» ou "Votre tâche est...» ou, tout simplement, "Je vous demande de... ». Les intervenants sont souvent mal à l'aise dans cette directivité.

La prescription des tâches entre les rencontres commence généralement en dernière partie de rencontre par un bref retour sur la situation à partir de ce que le client nous a apporté. Nous faisons ce retour sur un ton légèrement plus bas et avec un rythme plus lent. Dans le processus de changement, nous visons à faire faire un temps $\mathrm{d}^{\prime}$ arrêt dans la réaction du système-client. Celui-ci prend inconsciemment un recul, se distancie de l'émotion, ce qui facilite la construction d'une perception nouvelle ouverte au changement.

On établit ensuite la prochaine étape à atteindre. Si les possibilités de tâche n'ont pas encore été cherchées avec le client, on le fait, pour ensuite choisir une activité. II est important de prendre le temps d'amener le système-client à visualiser ce qu'il aura acquis lorsqu'il aura atteint cette étape. Notre expérience est que le client 
qui visualise l'apprentissage à atteindre de façon claire a déjà commencé intérieurement le changement, avant même de réaliser la tâche. Mais s'il ne peut le visualiser, il n'est pas prêt à s'engager, des obstacles sur le plan cognitif ou émotif I'en empêchant.

La tâche doit être la plus simple possible, comporter peu d'actions. On établit ses caractéristiques de façon détaillée: le temps, la durée, la fréquence, l'endroit, qui fait quoi et dans quel ordre, le rôle de chaque participant, qui fait le rappel de la tâche, quoi faire si un membre refuse de faire sa part. Il est important ici de "penser scénario", de voir les séquences d'actions dont chacune est importante mais est une partie d'un ensemble, de saisir leur enchaînement. Les actions doivent être normales, et elles peuvent être faites de façon spéciale ou même exagérée un peu (De Schazer, 1984, 1985, 1988).

Les règles sont établies par l'intervenant, non par les participants: c'est essentiel au succès de la tâche. Cela peut sembler limitatif, mais la fonction de la tâche spécifique est précisément d'encadrer l'action pour arriver à un apprentissage précis.

On vérifie de nouveau l'accord explicite de chaque participant et s'il a bien compris sa part. II est important que chaque membre du système-action soit impliqué; les tâches peuvent alors être identiques pour tous ou différentes et regroupées par sous-systèmes. On rappelle les avantages pour chacun, surtout dans les situations de conflits; on prévoit les obstacles possibles, et ce que chacun aura à faire pour les contourner. Lorsque les clients ont des craintes ou demeurent confus, il est utile de pratiquer, de faire un jeu de rôle, une simulation. Cela permet de rassurer les participants sur la faisabilité, d'identifier les obstacles non dits jusque-là, et d'y proposer une réponse.

Nous devons souligner un point important. L'équipe de Palo Alto (Fisch, 1981) insiste sur la nécessité paradoxale de «ralentir le client». Nous arrivons à la même conclusion avec les interventions directes. Il est préférable de prendre le temps de construire graduellement le changement par des tâches d'observation et de réflexion, avant de passer à des tâches d'action. Cela permet d'apprivoiser le changement, de construire un cadre mental où la situation nouvelle devient congruente à ce que la personne vit intérieurement ou dans ses habitudes. Cela fait découvrir les solutions avant de les expérimenter. Nous avons constaté que ce ralentissement ne ralentit pas l'intervention.

Voici un exemple de prescription de tâche:

" D'ici notre prochaine rencontre dans deux semaines, je vous demande de vous asseoir ensemble pour une période maximale de 10 minutes, deux fois par semaine, pour discuter 
de vos activités, de vos expériences, en respectant les règles de communication que nous avons travaillées aujourd'hui. Assurez-vous que vous avez des sujets positifs, pas uniquement des problèmes ou des insatisfactions.

Cela vous semble-t-il possible?

Quelles journées de la semaine pourrez-vous le faire?

À quel moment de la journée ? À quel endroit ?

Qui se charge de faire le rappel ?

Si vous ne pouvez le faire la journée prévue, vous le faites la journée suivante.

Il est important que vous vous limitiez à dix minutes pour éviter de retomber dans les patterns nuisibles de communication.

Après avoir exécuté la tâche, écrivez les satisfactions ou les difficultés que vous avez eues à respecter les façons de communiquer, les questions que vous avez. Nous les regarderons ensemble à la prochaine rencontre.

Avez-vous des questions par rapport à cette tâche?»

\section{Retour sur la tâche}

À la rencontre suivante, il est essentiel de vérifier l'exécution ou non de la tâche, généralement en début de l'entrevue. Le système-client peut tenter des diversions pour éviter ce retour lorsqu'il n'a pas exécuté la tâche. Si celle-ci a été faite, bravo; on explore comment ils ont réussi, ce qui a été facile et ce qui a été difficile, on clarifie les acquis et on renforce les gestes positifs, ce qui ouvre à l'étape suivante. II est alors important de bien faire le temps d'arrêt mentionné au début de la prescription, en résumant ce que nous constatons. Le ton un peu spécial ponctue et accentue les changements amorcés, et l'activité vers laquelle le système-client est guidé prend un sens particulier. Après la réalisation de la tâche, on fait un autre temps d'arrêt où on demande de commenter en détail ce qui s'est passé et ce qui a été acquis, pour l'intégrer à un niveau conscient.

Si la tâche a été remplie partiellement, on examine ce qui s'est passé, car il est possible qu'elle ait été remplie de façon valable.

Si elle n'a pas été remplie: on cherche l'erreur, qui se trouve habituellement de notre côté! Cette affirmation traumatise les intervenants, plus habitués de dire que les clients «résistent» ou «ne sont pas motivés ». Il s'agit d'une erreur d'input: ce qui a été proposé n'était pas recevable à ce moment ou de cette façon. 
Un retour sur le processus d'intervention permet de découvrir où se trouve l'erreur. La tâche a-t-elle été bien choisie? bien prescrite? a-t-elle été établie avec le client ou imposée? Respectait-elle les étapes du processus? Les participants étaient-ils vraiment d'accord ou avaient-ils une motivation différente que nous n'avons pas identifiée? Le contrat tenait-il compte de la demande de services? Le problème sur lequel nous avons pris une entente était-il bien choisi et bien reformulé? L'exploration était-elle suffisante? On commente ces points avec les participants, ce qui constitue un feed-back correctif.

Les tâches prescrites durant les rencontres suivent le même cheminement, mais habituellement $d^{\prime}$ une façon plus simple. Les tâches sont plus courtes: pas plus de quelques minutes. La principale différence est la présence du travailleur social; il peut apporter des correctifs, stimuler ou rassurer, toujours de façon brève pour ne pas diluer le processus du système-client. Ces scénarios sont très puissants pour aider le client à reconnaître ses capacités ignorées et à développer de la confiance. Elles servent aussi à identifier les motivations secrètes opposées, les craintes non révélées.

Pour l'intervenant, la tâche durant les entrevues lui permet de se retirer et de sortir de la triangulation lorsqu'elle devient un piège; elle lui permet aussi d'observer in vivo comment les personnes agissent ou interagissent, et comment elles réagissent lorsque le changement demandé commence à se réaliser.

Dans les tâches paradoxales, ou lorsque le client semble s'opposer, la prescription se fait différemment. Elle procède plutôt par suggestion, en prenant une position plus basse; on demande la permission, mentionne que l'idée peut sembler bizarre, mais qu'elle a pu être utile à d'autres personnes. Les verbes sont généralement conditionnels. Parfois les directives sont peu nombreuses: le choix se fait d'après la réaction du système-client. On peut se montrer $\mathrm{d}^{\prime}$ accord que la tâche est surprenante, mais en rappelant que la situation est difficile, et en se gardant bien de rassurer. Il est possible aussi de souligner qu'il ne s'agit que d'un essai.

Lors de la vérification à la rencontre suivante, si la tâche a été exécutée et apporte le résultat visé indirectement, nous félicitons les participants, tout en nous disant surpris, et maintenons I'approche paradoxale: nous ne savons pas ce qui a pu contribuer à un tel résultat, nous nous demandons ce qu'ils ont pu faire d'autre pour réussir ainsi et nous annonçons que la difficulté reviendra probablement. Si elle n'a pas été exécutée, habituellement le client a posé des gestes appropriés en lien avec le changement désiré, puisqu'il n'a pas maintenu le comportement problématique. Nous le soulignons 
« en passant», sans y attacher d'importance, et nous annonçons que cela ne durera pas, que la situation ne peut être changée si rapidement.

À ceux qui peuvent être choqués par cette démarche, nous rappelons que rien dans ce qui précède ne manque de respect au client (Néron et Gosselin, 1982), puisque la démarche répond à sa demande de service et que l'objectif désiré par le client a été atteint. De plus, ceux qui utilisent ces techniques savent qu'elles alternent constamment avec les interventions directes.

\section{Principales erreurs}

Ce sont :

- ne pas "penser scénario", ne pas reconnaître les séquences des actions et ne pas situer la fonction de la tâche dans le processus de changement;

- ne pas prendre le temps de prescrire correctement et le faire en vitesse, à la fin de la rencontre;

- demander une tâche trop complexe, donc ne pas évaluer la faisabilité ;

- ne pas respecter le rythme de changement du client et donner des tâches en bouchées trop grosses;

- céder aux pressions du client qui veut des résultats rapides;

- ne pas respecter le processus d'intervention dans le choix; par exemple, prescrire une tâche d'action au début.

\section{CONCLUSION}

L'utilisation systématique de tâches planifiées et structurées s'est révélée un instrument valable de changement. Elle constitue un temps fort dans les stratégies, et I'utilisation des autres techniques se termine bien dans une tâche, dont le résultat sert à son tour à préparer de façon plus efficace les autres techniques dans une continuité cohérente. Les tâches sont valables à toutes les étapes de l'intervention. Nous avons constaté que les changements visés sont atteints plus rapidement lorsque le client participe à l'élaboration des changements visés, qu'il se mobilise, et que l'observation et la réflexion avant de passer à l'action permettent d'apprivoiser des changements perçus d'abord comme difficiles. Les tâches sont 
particulièrement utiles lorsque le client n'ose plus continuer : le fait $\mathrm{d}^{\prime}$ agir contourne les craintes et les interdictions non conscientes au changement, et l'apprentissage vécu dans la tâche amène généralement à continuer.

Les recherches devront se faire sur les critères du choix des tâches, sur les modalités de prescription d'après les types et les situations ainsi que sur les ponts entre les tâches directes et les tâches indirectes. Nous avons confiance que la rigueur et la créativité des intervenants les amèneront à trouver des réponses et à concevoir des tâches innovatrices.

\section{Références bibliographiques}

De ShaZer, Steve (1984). Patterns of Brief Family Therapy. New York: Guilford Press.

De Shazer, Steve (1985). Keys to Solutions in Brief Therapy. New York: Norton.

De SHAZER, Steve (1988). Clues: Investigating Solutions in Brief Therapy. New York: Norton.

DU RANQUET, Mathilde (1992). Les approches en service social. SaintHyacinthe: Edisem-Vigot, $2^{\mathrm{e}}$ édition.

EPSTEIN, Laura (1980). Helping People: The Task-Centered Approach. Saint Louis: C. Mosby.

FISCH, Richard, John WEAKLAND et Lynn SEGAL (1981). Les tactiques du changement. Paris: Seuil.

FISHER, Stuart (1980). "The Use of Time-Limits in Brief Psychotherapy: a Comparison of Six-session, Twelve-session and Unlimited Treatment with Families », Family Process, vol. 19: 377-392.

Fortune, Anne, ed. (1985). Task-Centered Practice with Families and Groups. New York: Springer.

HALEY, Jay (1974). Nouvelles stratégies en thérapie familiale. Montréal: Éd. France-Amérique.

HALEY, Jay (1986). Un thérapeute hors du commun, Milton Erickson. Paris: Seuil.

MAPLE, Frank (1988). L'entrevue dynamique. Québec: Éd. St-Yves.

MINUCHIN, Salvador et Braulio MONTALVO (1966). "An Approach for Diagnosis of the Low Socio-Economic Families», Psychiatry.

MINUCHIN, Salvador et Charles FISHMAN (1981). Family Therapy Techniques. Cambridge: Harvard University Press.

NÉRON, Isidore et Raynald GOSSELIN (1982). "Les interventions stratégiques: une pratique discutable», Service social, vol. 31, n 2-3.

PANNETON, Marthe (1992). "Les interventions stratégiques avec les familles recomposées", Le travailleur social, vol. 60, $\mathrm{n}^{\circ} 2$. 
REID, William (1985). Family Problem Solving. New York: Columbia University Press.

ReID, William (1992). Task Strategies. New York: Columbia University Press.

REID, William et Laura EPSTEIN (1972). Task-Centered Casework. New York: Columbia University Press.

REID, William et Laura EPSTEIN, ed. (1977). Task-Centered Practice. New York: Columbia University Press.

WELLS, Richard (1994). Planned Short-Term Treatment. New York: Free Press. 